\title{
Pay attention to the biological nanoparticles when studying the protein corona on nanomedicines
}

Simonsen, Jens B.; Münter, Rasmus Dithmar

Published in:

Angewandte Chemie International Edition

Link to article, DOI:

10.1002/anie.202004611

Publication date:

2020

Document Version

Peer reviewed version

Link back to DTU Orbit

Citation (APA):

Simonsen, J. B., \& Münter, R. D. (2020). Pay attention to the biological nanoparticles when studying the protein corona on nanomedicines. Angewandte Chemie International Edition, 59(31), 12584-12588.

https://doi.org/10.1002/anie.202004611

\section{General rights}

Copyright and moral rights for the publications made accessible in the public portal are retained by the authors and/or other copyright owners and it is a condition of accessing publications that users recognise and abide by the legal requirements associated with these rights.

- Users may download and print one copy of any publication from the public portal for the purpose of private study or research.

- You may not further distribute the material or use it for any profit-making activity or commercial gain

- You may freely distribute the URL identifying the publication in the public portal

If you believe that this document breaches copyright please contact us providing details, and we will remove access to the work immediately and investigate your claim. 


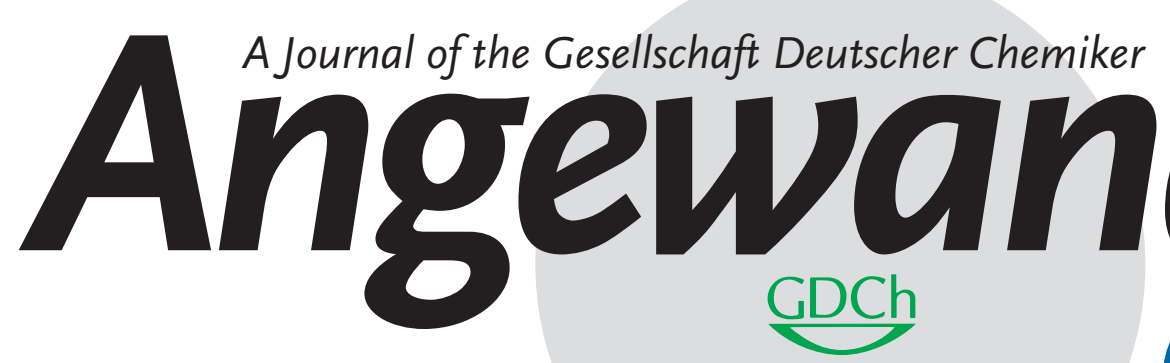

International Edition

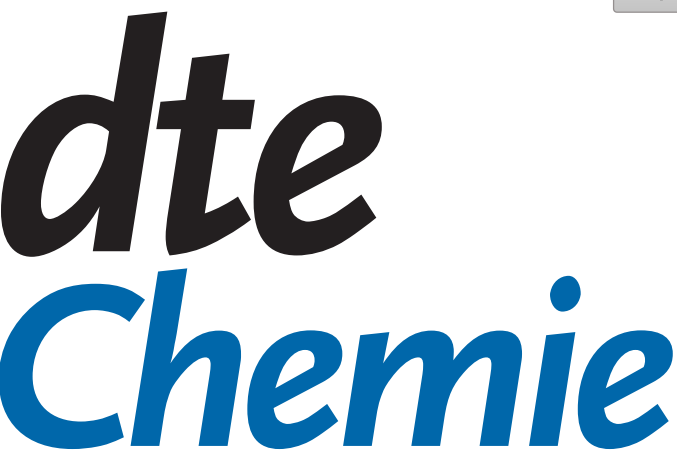

www.angewandte.org

\section{Accepted Article}

Title: Pay attention to the biological nanoparticles when studying the protein corona on nanomedicines

Authors: Jens B Simonsen and Rasmus Münter

This manuscript has been accepted after peer review and appears as an Accepted Article online prior to editing, proofing, and formal publication of the final Version of Record (VoR). This work is currently citable by using the Digital Object Identifier (DOI) given below. The VoR will be published online in Early View as soon as possible and may be different to this Accepted Article as a result of editing. Readers should obtain the VoR from the journal website shown below when it is published to ensure accuracy of information. The authors are responsible for the content of this Accepted Article.

To be cited as: Angew. Chem. Int. Ed. 10.1002/anie.202004611

Link to VoR: https://doi.org/10.1002/anie.202004611 


\title{
Pay attention to the biological nanoparticles when studying the protein corona on nanomedicines
}

\author{
Jens B. Simonsen ${ }^{1, *}$ and Rasmus Münter ${ }^{1}$ \\ ${ }^{1}$ Department of Health Technology, Technical University of Denmark, 2800 Kgs. Lyngby, \\ Denmark
}

Email: jbak@dtu.dk

\begin{abstract}
:
The protein corona of nanoparticles has in recent years received considerable attention, and even been postulated to be the missing link in the translation of nanomedicines from benchtop to bedside. Here, we highlight the different types of biological nanoparticles present in blood that need to be considered in the protein corona research field. We map their size, density and plasma concentrations and use this information to stress potential challenges related to the isolation of nanomedicines, with particular focus on liposomes, when using the traditional isolation methods that separate according to size and density.
\end{abstract}

The protein corona is currently a hot topic in the field of nanomedicine. It refers to the adsorption of serum proteins onto nanoparticles during blood circulation. This type of remodeling of nanomedicines (nanoparticle-based drugs and drug delivery systems) is thought to have an essential impact on their targeting properties, biological fate and thus therapeutic efficacy ${ }^{[1,2]}$ Among the most studied nanomedicines in the framework of protein corona, and clinically most successful nanomedicines, are polyethylene glycol (PEG)-coated liposomes, so-called stealth liposomes. ${ }^{[3]}$ It was originally proposed that the long circulating time of liposomes with PEG grafting was due to the hydrophilic polymer reducing the binding of opsonins (proteins that aid immune cells in recognizing and clearing foreign particles) onto the liposomal surface. ${ }^{[4,5]}$ Conversely, it could also be argued that specific proteins ("dysopsonins") binding to PEG chains might contribute to the stealth-like properties of PEGylated liposomes in blood. ${ }^{[6,7]}$ Along these lines, the reported amount of proteins on PEGylated liposomes, in terms of the protein binding value $\mathrm{P}_{\mathrm{B}}$, defined as gram (protein) per mol (liposome lipids), surprisingly, varies a lot across studies, ranging from approx. 5 to 120 $\mathrm{g} / \mathrm{mol}$ in vitro in mouse plasma, ${ }^{[1,8-11]}$ and even $>1000 \mathrm{~g} / \mathrm{mol}$ in vivo in humans ${ }^{[12]}$. Hence, we cannot clearly conclude from these studies, whether the long circulation time of PEGylated liposomes is due to reduced or increased protein binding - or put in another way, whether PEGylated liposomes are coated with a protein corona or not. What could possibly explain these very different outcomes and conclusions reported about the protein corona on PEGylated liposomes? Maybe there are some underlying inherent components that we need to consider when studying the protein corona on PEGylated liposomes and any other type of nanomedicines? 
In this viewpoint, we will take a detailed look at the endogenous (biological) nanoparticles present in blood and serum, herein referred to as bio-nanoparticles. Next, we will discuss how such bio-nanoparticles could affect assessment of protein adsorption to nanomedicines, with focus on liposomes. We also briefly touch upon how the commonly used methods for studying the protein corona can reveal the presence of bio-nanoparticle contaminants and thereby assess if the bio-nanoparticle contamination is a concern for a specific setup. It should be stressed that the protein corona that is typically being studied, and is also discussed here, is the so-called "hard" protein corona. The hard protein corona refers to the proteins that stay associated with the nanoparticle after isolation from plasma, whereas the "soft" corona refers to proteins that dissociate during the purification steps.

\section{The bio-nanoparticles in blood}

The bio-nanoparticles in blood/serum include extracellular vesicles (EVs) and lipoproteins. Extracellular vesicles (EVs) are small particles released by all types of cells into all kinds of body fluids, including blood. ${ }^{[13]}$ EVs are suggested to be implicated in a wide range of physiological and pathological processes, including cancer and heart diseases, by mediating intercellular communication, and through their proposed delivering capabilities. ${ }^{[14]}$ EVs are due to their different origin a very heterogeneous class of nanoparticles, spanning from 30$1000 \mathrm{~nm}$ in size, with the majority being $<150 \mathrm{~nm}$ in diameter. ${ }^{[15]}$ These bio-nanoparticles are like liposomes comprised of a lipid-bilayer surface. In addition, EVs contain both membranebound proteins and water soluble proteins in their aqueous core.

Lipoproteins are also a very heterogeneous family of particles that includes five different subspecies defined by their density: ${ }^{[16]}$ High-density lipoproteins (HDL), low-density lipoproteins (LDL), intermediate-density lipoproteins (IDL), very low-density lipoproteins (VLDL), and, with lowest density, chylomicrons (CMs). One of the most important functions of lipoproteins is to deliver lipid cargo throughout the body, including cholesterol esters and triglycerides. To make these hydrophobic lipids 'soluble' in body fluids such as blood, they are packed into the core of the lipoproteins and surrounded by a monolayer of amphipathic phospholipids. In addition, amphipathic apolipoproteins function as a structural scaffold for the lipoprotein particle, and are involved in the recognition of cells as well as transport of the hydrophobic cargo into cells. The apolipoprotein apoA-I is mostly associated with HDL and accounts for almost $70 \%$ of the mass of HDL protein content. ApoB-100 is associated with LDL, IDL, and VLDL, whereas apoB-48 is uniquely associated with CMs. Figure $1 \mathrm{~A}$ shows the size and density for both EVs, ${ }^{[17]}$ the different classes of lipoproteins, ${ }^{[17]}$ and liposomes, while figure 1B shows the expected plasma concentrations of the corresponding nanoparticles. ${ }^{[18]}$ 
A

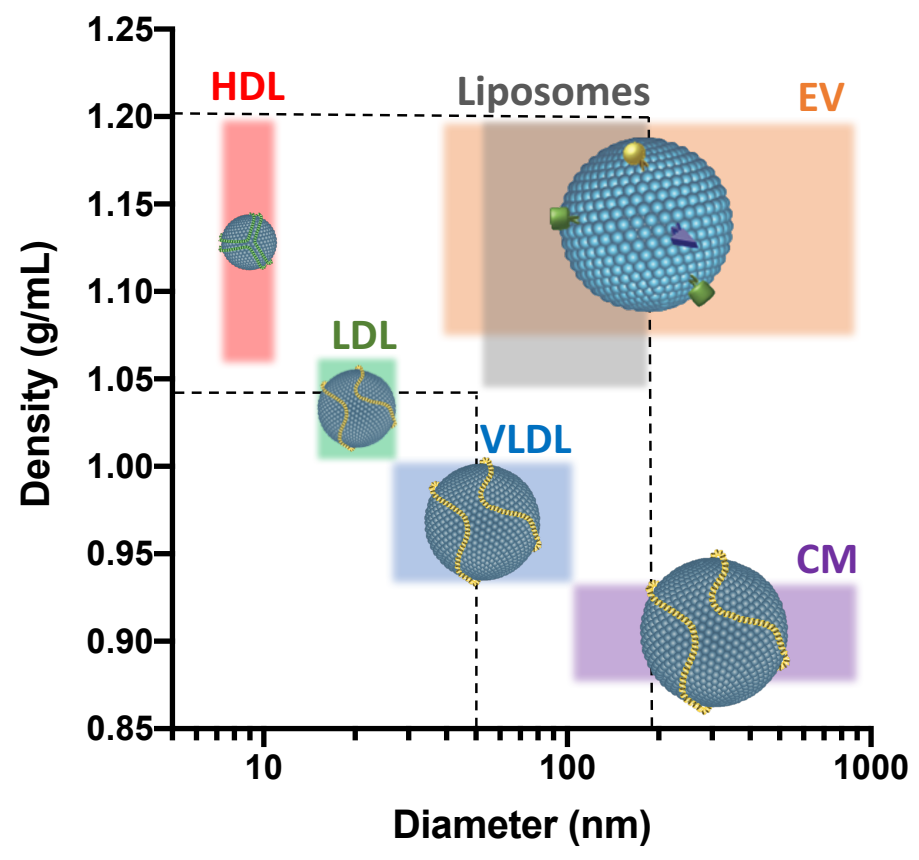

B

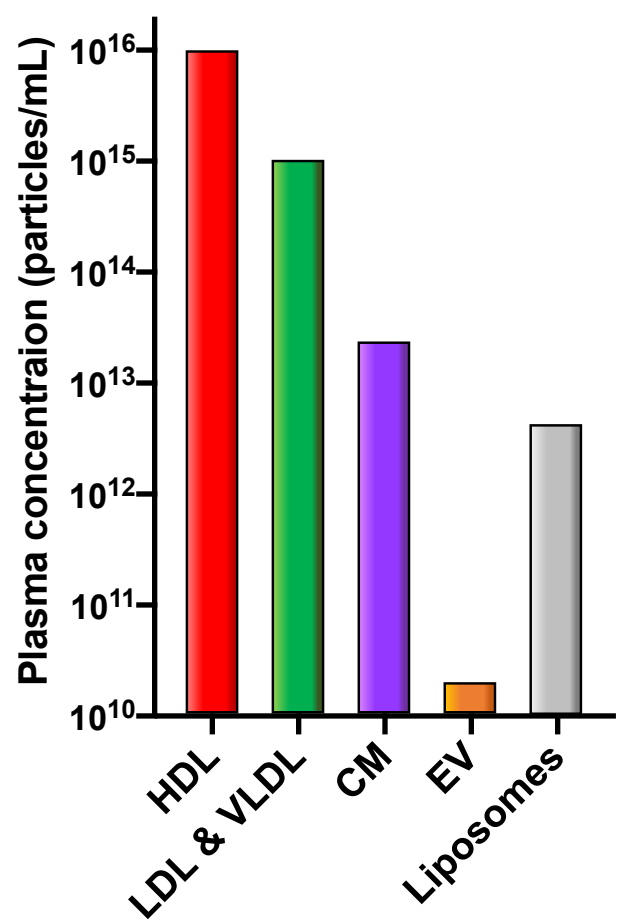

Figure 1. (A) A representation of the biophysical properties of extracellular vesicles (EVs); the different lipoprotein classes: high density lipoprotein (HDL), low-density lipoprotein (LDL), very low-density lipoprotein (VLDL), chylomicron (CM); and liposomes. ${ }^{[17]}$ Liposome density will depend on the protein adsorption, ${ }^{[19]}$ with a $10 \mathrm{~nm}$ thick dense layer of proteins on a $100 \mathrm{~nm}$ liposome resulting in a density of $\sim 1.2 \mathrm{~g} / \mathrm{mL}$, whereas both empty and drug loaded liposomes have a density around $1.05 \mathrm{~g} / \mathrm{mL}$. ${ }^{[0]}$ The mass density and size intervals of the different types of nanoparticles are depicted by colored boxes. The bio-nanoparticles contain a phospholipid surface (blue color) in addition to apolipoproteins (green, yellow) on lipoproteins and other type of proteins on EVs. (B) Particle concentration of the different types of bio-nanoparticles in human plasma. ${ }^{[17,18]}$ The liposome particle concentration is calculated for $1 \mathrm{mM}$ lipid based on the assumption that liposomes are $100 \mathrm{~nm}$ with an average lipid headgroup area of $0.425 \mathrm{~nm}^{2}{ }^{[21]}$

The estimated particle concentrations of HDL $\left(9 \times 10^{15} / \mathrm{mL}\right), \mathrm{LDL}\left(10^{15} / \mathrm{mL}\right.$ including VLDL and $I D L)$, and $C M s\left(2 \times 10^{13} / \mathrm{mL}\right)$ in plasma, are based on an average of three apoA-I per HDL and one apoB per LDL and CM, respectively. ${ }^{[17,18,22]}$ The well-defined number of apolipoproteins per particle makes it possible to determine their particle concentrations based on ELISA measurements. Opposite to the lipoproteins, the particle concentration of EVs is difficult to assess, as no universal biomarker molecule has been identified for the EV population. We recently estimated the EV concentration in plasma to be $\sim 10^{10} / \mathrm{mL}$, based on a thorough literature survey ${ }^{[18]}$ keeping in mind that the methods used to enumerate EVs suffer from sensitivity and specificity. While EVs are a minority population in the plasma of healthy humans their abundance increase significantly in cancer patients. ${ }^{[23]}$ This may be worth considering when studying the protein corona on nanomedicines in blood from cancer animal models and cancer patients. It is clear from Figure 1B that the liposomes, at the (clinically relevant) concentrations used for protein corona studies, is a minor nanoparticle population 
in the total pool of nanoparticles in plasma, also when only focusing on nanoparticles with a size around $100 \mathrm{~nm}$ : the typical size of the liposomes used in the clinic. ${ }^{[3]}$

\section{Co-isolation of bio-nanoparticles with liposomes}

Taking the facts presented in Figure 1 into account, it becomes apparent that the size- and density-based isolation methods that are frequently used in the protein corona field, can potentially lead to a significant amount of co-isolated bio-nanoparticles in liposome isolates.

Size-exclusion chromatography (SEC) separates according to size and is one of the most commonly used methods to isolate liposomes from plasma. Even when applying standard SEC columns such as Sepharose CL-4B with a cut-off value around $20-40 \mathrm{~nm}$, that should remove the highly abundant HDL and LDL, the large-size populations of VLDL, EVs, and CMs will still co-elute with the $100 \mathrm{~nm}$ liposomes. An additional consideration is that too much material loaded onto the column can lead to spillover of e.g. LDL particles into the liposome fraction, why sample size should be considered carefully. Due to the overlapping size distributions of liposomes and bio-nanoparticles, asymmetric flow-field-flow fractionation (AF4) ${ }^{[24]}$ does unfortunately not circumvent this issue. One way to minimize the $\mathrm{CM}$ concentration in plasma is to use fasting blood. ${ }^{[25]}$ Another approach could however be to remove bio-nanoparticles from the plasma before adding the liposomes, using a similar purification method as that applied to isolate the liposomes. ${ }^{[26]}$ Centrifugation is also prone to lipoprotein contamination because the sedimentation rate of spherical objects at constant acceleration depends on both the difference between the density of the particle and the density of the medium, as well as on the square of the particle radius, according to Stokes' law. Thus, if we assume that the density of LDL and empty liposomes are similar, the sedimentation rate of $100 \mathrm{~nm}$ liposomes is $\approx 16 \times$ faster than $25 \mathrm{~nm}$-sized LDL. However, because the LDL concentration is more than 16 times higher than the expected level of liposomes in blood (see Figure 1B), the final centrifugation product (assumed to contain liposomes) may, in addition to EV contamination, eventually contain a significant amount of LDL. The CMs will, opposite to e.g. LDLs, move towards the top due to having a density lower than that of water, and hence pose a minor issue in centrifugation-based setups. Further, some liquid spillover into the pellet is hard to avoid during the handling. In summary, some of the classical methods used to isolate liposomes from plasma/serum do, according to the arguments above, probably contain bionanoparticle contamination.

\section{Does the co-isolation of bio-nanoparticles pose a problem to the assessment of the protein corona on liposomes?}

To answer this question, we need to understand the composition of the bio-nanoparticles. As mentioned before, EVs contain membrane bound and water soluble proteins, while the specific classes of lipoproteins contain specific apolipoproteins. In addition to the apoA-I and ApoB proteins, other types of apolipoproteins (apoC, apoD, apoM etc.), ${ }^{[16]}$ as well as many types of non-apolipoproteins, are also reported to be associated with lipoproteins including HDL. ${ }^{[27]}$ Taken together, we start to appreciate that the proposed bio-nanoparticle contamination in liposome isolates from plasma and serum, most likely gives rise to a significant amount of bio-nanoparticle-associated proteins in addition to the proteins associated with the liposomes. The presence of bio-nanoparticles in liposome isolates would 
thus add to the measured protein (and lipid) content in the sample, and will in that case skew the results and conclusions about the degree of protein adsorption to liposomes. This challenge also applies to other types of nanomedicines that possess similar physical properties as the bio-nanoparticles and are isolated from plasma using similar methodology.

\section{Can standard methods used to characterize the protein corona on liposomes asses the presence of bio-nanoparticle contamination?}

The most commonly used technique for quantifying the protein level in liposome isolates post blood/serum exposure is the bicinchoninic acid assay $(B C A) \cdot{ }^{[9,12]}$ This or similar assays for determination of protein concentrations does however not distinguish if the proteins in a sample are bound to the isolated liposomes, or are just co-eluting/-precipitating with the liposomes. Running a blank plasma sample without liposomes (control sample), ${ }^{[1,10]}$ can however be used to assess if bio-nanoparticle contamination is an issue. As the presence of a protein corona should give rise to an increased protein content in the isolate when liposomes are added to the plasma, the increase over the background can be used to calculate the true protein corona content.

The identity of the specific proteins isolated can also be used to assess if any background detected in a liposome-free blank plasma sample is due to lipoprotein contaminants. Sodium dodecyl sulfate-polyacrylamide gel electrophoresis (SDS-PAGE) and mass spectrometry (MS) are often used to study the protein composition in isolated liposome samples. These methods are different when it comes to their qualitative and quantitative assessments. MS proteomics have very high sensitivity, and MS thus allows for identification of many different proteins in a sample. From a qualitative point of view, membrane-spanning proteins are a strong indicator on co-isolated EVs. However, it can be difficult to assess the quantitative value of MS studies when no proper references are included. While SDS-PAGE is not as sensitive and specific as MS approaches, this type of data provide valuable semi-quantitative information, when comparing to protein references. If the apolipoprotein content is similar in a liposomefree and liposome-containing plasma sample after centrifugation or chromatography, lipoprotein contaminants are most likely an issue. Apolipoproteins are indeed often reported

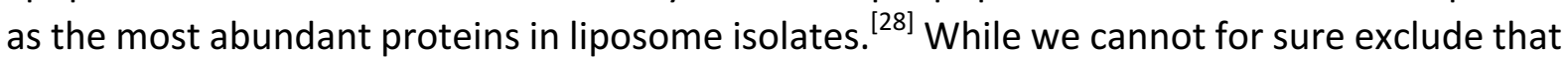
these truly bind to the liposomes (apoA, apoC and apoE are known as exchangeable apolipoproteins, ${ }^{[29]}$ while both apoB variants are associated with the same entity during its circulation), only an increase in concentration for a given apolipoprotein in the isolate, when comparing a liposome-free to a liposome-containing plasma sample, can determine if the apolipoproteins truly associate to the liposomes.

Another popular technique for characterizing liposome isolates post blood/serum exposure, is transmission electron microscopy (TEM). In negative stain TEM, liposomes typically collapse into cupped shaped structures due to the dehydration of the liposomes during the preparation steps. This feature also applies to EVs, and it can thus be difficult to distinguish liposomes from EVs. Lipoproteins do however not collapse due to their hydrophobic core, and circular structures can hence indicate the presence of lipoproteins. 
Dynamic light scattering (DLS) is often used to determine the size of the particles in isolates after SEC of AF4, ${ }^{[1,12,24]}$ and can under some circumstances also reveal the presence of bionanoparticle contaminants. Whereas DLS is a strong technique for studying mono disperse samples like well-defined liposomes, it is however challenged when studying samples containing several distinct particle size distributions. As larger particles scatter light more efficient than small, DLS measurements favor larger particles when presenting the data weighted by scatter intensity, thus challenging the detection of LDL and HDL contaminants. If a significant feature appears around $25 \mathrm{~nm}$ or $50 \mathrm{~nm}$ - and not $100 \mathrm{~nm}$ in the DLS data weighted by scatter intensity - the sample likely contains way more LDL or VLDL, respectively, than $100 \mathrm{~nm}$-sized liposomes. DLS is in addition often used to report on the thickness of the protein corona. ${ }^{[30-32]}$ - and in other cases finding that liposomes shrink in plasma. ${ }^{[1,12]}$ Bionanoparticles can however also pose an issue for determining the presence and thickness of a protein corona, as the technique cannot discriminate between liposomes and similar-sized particles such as EVs and CMs. As shown in figure 1, both EVs and CMs have broad sizedistributions, with some being larger than the typical drug delivery liposomes. When it, using DLS, has been found that the protein corona of liposomes has a thickness of $40-50 \mathrm{~nm}{ }^{[31]}$ this observation could hence also be explained by $\mathrm{CM}$ and EV contaminations in the sample. This pose an even more severe challenge, when running the experiment in situ and hence not performing a separation-step beforehand. ${ }^{[30-32]}$ Running a liposome-free sample is thus also of utmost importance for such size-measurements as well.

Nanoparticle tracking analysis (NTA) is a technique that is commonly used in the EV research to determine the EV concentration and their size distribution. NTA can in principle circumvent the issues related with DLS, as NTA measures the size of individual nanoparticles and can potentially distinguish liposomes from other nanoparticle species when using a fluorescent label on the liposomes. To add to the complexity, we however recently reported that many commercially available lipid-anchored fluorophores (typically used for tracking and visualizing liposomes) have a propensity to dissociate from liposomes into plasma components ${ }^{[33]}$ probably into bio-nanoparticles that contain a large pool of phospholipid surfaces. Importantly, this means that bio-nanoparticle contamination may also affect experimental setups relying on fluorescent labels.

\section{Conclusions and final remarks}

We hope this viewpoint brings awareness of bio-nanoparticles and how these may pose a challenge to the study and quantification of the protein corona on liposomes and other types of nanomedicines. This aspect could to some extent explain why it has been so challenging to quantify the protein adsorption to PEGylated liposomes, and why the reported values differ so much. That said, it should also be stressed that other entities beyond bio-nanoparticles such as protein aggregates ${ }^{[34]}$ - can pose a contamination issue, similarly skewing the results on measured protein corona content. We have here briefly discussed how some of the traditional methods used to characterize the protein corona can be used to verify and quantify the presence of bio-nanoparticles (in particular lipoproteins) and thereby assess if bio-nanoparticle contamination is an issue. It should be mentioned that other less commonly 
used methods have been presented in literature, which instead of separation based on physical parameters (size and density) are based on chemical modifications such as magnetism ${ }^{[35]}$ or click chemistry, ${ }^{[36]}$ or immuno-labelling with detection based on electron microscopy ${ }^{[37]}$ or flow cytometry. ${ }^{[38]}$ Such methods are not as commonly used as SEC and centrifugation, partly due to technical limitations such as challenges with encapsulation of magnetic particles and limited sensitivity of commercial flow cytometers. These alternative methods could, however, potentially circumvent bio-nanoparticle contamination issues.

In addition to the protein corona quantification, the downstream assessment of the properties (incl. targeting) of liposomes/nanomedicines post plasma/serum exposure could be challenged if a significant amount of bio-nanoparticles is co-isolated with the liposomes. Along this line, if labile fluorophores with a propensity to dissociate from liposomes into bionanoparticles $^{[33]}$ have been used, these could also challenge the interpretation of fluorescence-based uptake studies.

Hopefully, this comment will start a constructive discussion about whether improvements are needed for the quantitative protein corona assessment. To this end, we would like to stress the importance to include proper controls to justify the true amount and the specific proteins that comprise the protein corona on the recovered nanomedicine, e.g. by subjecting control plasma/serum to the same purification procedures as the nanomedicine-containing samples. ${ }^{[1,10]}$ In case the protein content in such a nanomedicine-free sample is relatively high and/or the nanomedicine concentration is low, the background protein content could account for a significant amount of the protein corona in the 'apparent' nanomedicine isolate.

Finally, we encourage EV and protein corona scientists to share their knowledge across these two similar disciplines when it comes to the methods used to isolate and characterize vesicular nanoparticles from plasma and serum. With these strategies and efforts, we may soon be able to design better nanomedicines that either are fully resistant to plasma components or facilitate the association of specific plasma components to improve the circulation or specific targeting properties, respectively, and thereby improve the overall therapeutic efficacy of the future nanomedicines.

\section{References}

[1] M. Hadjidemetriou, Z. Al-Ahmady, M. Mazza, R. F. Collins, K. Dawson, K. Kostarelos, ACS Nano 2015, 9, 8142-8156.

[2] C. D. Walkey, W. C. W. Chan, Chem. Soc. Rev. 2012, 41, 2780-2799.

[3] U. Bulbake, S. Doppalapudi, N. Kommineni, W. Khan, Pharmaceutics 2017, 9, 12.

[4] A. L. Klibanov, K. Maruyama, V. P. Torchilin, L. Huang, FEBS Lett. 1990, 268, 235-237.

[5] A. Mori, A. L. Klibanov, V. P. Torchilin, L. Huang, FEBS Lett. 1991, 284, 263-266.

[6] S. M. Moghimi, J. Szebeni, Prog. Lipid Res. 2003, 42, 463-478.

[7] S. Schöttler, G. Becker, S. Winzen, T. Steinbach, K. Mohr, K. Landfester, V. Mailänder, F. R. Wurm, Nat. Nanotechnol. 2016, 11, 372-377. 
[8] S. C. Semple, A. Chonn, P. R. Cullis, Adv. Drug Deliv. Rev. 1998, 32, 3-17.

[9] N. Dos Santos, C. Allen, A.-M. Doppen, M. Anantha, K. A. K. Cox, R. C. Gallagher, G. Karlsson, K. Edwards, G. Kenner, L. Samuels, et al., Biochim. Biophys. Acta - Biomembr. 2007, 1768, 1367-1377.

[10] K. Kristensen, T. B. Engel, A. Stensballe, J. B. Simonsen, T. L. Andresen, J. Control. Release 2019, 307, 1-15.

[11] M. Hadjidemetriou, Z. Al-Ahmady, K. Kostarelos, Nanoscale 2016, 8, 6948-6957.

[12] M. Hadjidemetriou, S. McAdam, G. Garner, C. Thackeray, D. Knight, D. Smith, Z. AlAhmady, M. Mazza, J. Rogan, A. Clamp, et al., Adv. Mater. 2019, 31, 1803335.

[13] G. Raposo, W. Stoorvogel, J. Cell Biol. 2013, 200, 373-383.

[14] Y. Yuana, A. Sturk, R. Nieuwland, Blood Rev. 2013, 27, 31-39.

[15] E. van der Pol, A. N. Böing, E. L. Gool, R. Nieuwland, J. Thromb. Haemost. 2016, 14, 48-56.

[16] J. E. Vance, D. E. Vance, Biochemistry of Lipids, Lipoproteins and Membranes, Elsevier, 2008.

[17] J. B. Simonsen, Circ. Res. 2017, 121, 920-922.

[18] K. B. Johnsen, J. M. Gudbergsson, T. L. Andresen, J. B. Simonsen, Biochim. Biophys. Acta Rev. Cancer 2019, 1871, 109-116.

[19] N. S. De Silva, C. H. Siu, J. Biol. Chem. 1981, 256, 5845-5850.

[20] D. Mehn, P. Iavicoli, N. Cabaleiro, S. E. Borgos, F. Caputo, O. Geiss, L. Calzolai, F. Rossi, D. Gilliland, Int. J. Pharm. 2017, 523, 320-326.

[21] Y. Wang, P. Gkeka, J. E. Fuchs, K. R. Liedl, Z. Cournia, Biochim. Biophys. Acta-Biomembr. 2016, 1858, 2846-2857.

[22] P. M. Hutchins, G. E. Ronsein, J. S. Monette, N. Pamir, J. Wimberger, Y. He, G. M. Anantharamaiah, D. S. Kim, J. E. Ranchalis, G. P. Jarvik, Clin. Chem. 2014, DOI 10.1373/clinchem.2014.228114.

[23] A. Caivano, I. Laurenzana, L. De Luca, F. La Rocca, V. Simeon, S. Trino, F. D’Auria, A. Traficante, M. Maietti, T. Izzo, et al., Tumor Biol. 2015, 36, 9739-9752.

[24] C. Weber, J. Simon, V. Mailänder, S. Morsbach, K. Landfester, Acta Biomater. 2018, 76, $217-$ 224.

[25] K. Nakajima, T. Nakano, Y. Tokita, T. Nagamine, A. Inazu, J. Kobayashi, H. Mabuchi, K. L. Stanhope, P. J. Havel, M. Okazaki, et al., Clin. Chim. Acta 2011, 412, 1306-1318.

[26] K. Yang, B. Mesquita, P. Horvatovich, A. Salvati, Acta Biomater. 2020, 106, 314-327.

[27] A. S. Shah, L. Tan, J. L. Long, W. S. Davidson, J. Lipid Res. 2013, 54, 2575-2585.

[28] L. Digiacomo, F. Cardarelli, D. Pozzi, S. Palchetti, M. A. Digman, E. Gratton, A. L. Capriotti, M. Mahmoudi, G. Caracciolo, Nanoscale 2017, 9, 17254-17262.

[29] M. C. Phillips, J. Lipid Res. 2013, 54, 2034-2048.

[30] F. Giulimondi, L. Digiacomo, D. Pozzi, S. Palchetti, E. Vulpis, A. L. Capriotti, R. Z. Chiozzi, A. Laganà, H. Amenitsch, L. Masuelli, et al., Nat. Commun. 2019, 10, 3686.

[31] V. Colapicchioni, M. Tilio, L. Digiacomo, V. Gambini, S. Palchetti, C. Marchini, D. Pozzi, S. Occhipinti, A. Amici, G. Caracciolo, Int. J. Biochem. Cell Biol. 2016, 75, 180-187. 
[32] G. Caracciolo, S. Palchetti, L. Digiacomo, R. Z. Chiozzi, A. L. Capriotti, H. Amenitsch, P. M. Tentori, V. Palmieri, M. Papi, F. Cardarelli, et al., ACS Appl. Mater. Interfaces 2018, 10, 22951-22962.

[33] R. Münter, K. Kristensen, D. Pedersbæk, J. B. Larsen, J. B. Simonsen, T. L. Andresen, Nanoscale 2018, 10, 22720-22724.

[34] C. Weber, S. Morsbach, K. Landfester, Angew. Chemie Int. Ed. 2019, 58, 12787-12794.

[35] C. P. S. Tilcock, E. F. Sommerman, R. S. Molday, P. R. Cullis, J. Liposome Res. 1988, 1, 137150.

[36] R. Pattipeiluhu, S. Crielaard, I. Klein-Schiphorst, B. I. Florea, A. Kros, F. Campbell, ACS Cent. Sci. 2020, DOI 10.1021/acscentsci.9b01222.

[37] P. M. Kelly, C. Åberg, E. Polo, A. O’Connell, J. Cookman, J. Fallon, Ž. Krpetić, K. A. Dawson, Nat. Nanotechnol. 2015, 10, 472-479.

[38] C. Weber, M. Voigt, J. Simon, A.-K. Danner, H. Frey, V. Mailänder, M. Helm, S. Morsbach, K. Landfester, Biomacromolecules 2019, 20, 2989-2999. 